\title{
CONSOLIDACIÓN LEGAL Y REGULATORIA DEL SERVICIO PÚBLICO DE LAS COMUNICACIONES EN COLOMBIA 1847-2020 ${ }^{1}$
}

\author{
LEGAL AND REGULATORY CONSOLIDATION OF THE PUBLIC \\ COMMUNICATIONS SERVICE IN COLOMBIA 1847-2020
CONSOLIDAÇÃO JURÍDICA E REGULAMENTAR DO SERVIÇO DE COMUNICAÇÕES PÚBLICAS NA COLÔMBIA 1847-2020

\author{
Raúl Andrés Tabarquino Muñoz
}

Doctor en Administración, Universidad del Valle. Director e Investigador del Grupo Gestión y Políticas Públicas, Categoría A de Colciencias; Coordinador del Doctorado en Gobierno, Política Pública y Administración Pública; Docente Tiempo Completo Departamento de Administración y Organizaciones, Universidad del Valle. ORCiD: 0000-0002-7866-1875. E-mail: raul.tabarquino@correounivalle.edu.co, Colombia.

DOI: https://doi.org/10.22267/rtend.222301.195

\footnotetext{
${ }^{1}$ Este artículo de investigación es producto del desarrollo de la línea de investigación en servicios públicos del grupo de Gestión y Políticas Públicas (Categoría A) de la Universidad del Valle; recopila los resultados y análisis de la tesis doctoral en Administración del profesor Tabarquino denominada "La Red de política pública de regulación del servicio público de las tecnologías de la información y las comunicaciones (TIC) en Colombia 2009-2016: El Acceso (Universalidad) y La Equidad como Principios Rectores".
} 


\title{
Resumen
}

El objetivo es precisar la consolidación legal y regulatoria de las comunicaciones en Colombia como servicio público entre los años 1847-2020. La metodología se basa en un enfoque mixto información primaria y secundaria. Los resultados evidencian que la estructura legal y regulatoria del servicio público de las comunicaciones en Colombia entre los años 1847 y 2020, a pesar de las diferentes ideologías y toma decisiones, responden a la premisa de aprendizaje institucional con alianzas y transferencia de conocimiento técnico por parte de actores internacionales y privados. La conclusión está enmarcada en un sector dinámico y progresivo, que a pesar que se regule como servicio público es muy diverso y complejo por la incidencia de la regulación internacional.

Palabras clave: comunicaciones; estado; regulación; servicio público; TIC.

JEL: B41; D4; H11; L8; L51

\begin{abstract}
The objective is to specify the legal and regulatory consolidation of communications in Colombia as a public service between the years 1847-2020. The methodology is based on a mixed approach - primary and secondary information. The results show that the legal and regulatory structure of the public communications service in Colombia between 1847 and 2020, despite the different ideologies and decision-making, respond to the premise of institutional learning with alliances and transfer of technical knowledge by of international and private actors. The conclusion is marked in a dynamic and progressive sector, which despite being regulated as a public service is very diverse and complex due to the incidence of international regulation.
\end{abstract}

Keywords: communications; regulation; state; public service; TIC.

JEL: B41; D4; H11; L8; L51 


\section{Resumo}

O objetivo é especificar a consolidação legal e regulatória das comunicações na Colômbia como um serviço público entre os anos 1847-2020. A metodologia é baseada em uma abordagem mista - informações primárias e secundárias. Os resultados mostram que a estrutura jurídica e regulatória do serviço público de comunicações na Colômbia entre 1847 e 2020, apesar das diferentes ideologias e tomadas de decisão, respondem à premissa de aprendizagem institucional com alianças e transferência de conhecimento técnico de atores internacionais e privados. . A conclusão é marcada em um setor dinâmico e progressivo, que apesar de ser regulado como serviço público é muito diversificado e complexo devido à incidência de regulação internacional.

Palavras-chave: comunicações; estado; regulação; serviço público; TIC.

JEL: B41; D4; H11; L8; L51

\section{Introducción}

La disertación investigativa, legal y regulatoria de los servicios públicos es importante, porque es un proceso inherente a las finalidades del Estado por su relación directa con el bienestar social y el contexto político-social en cualquier territorio (Hiriart et al. 2004; Rodríguez, 2013; Rozas y Hantke, 2013; Spiller y Abdala, 2000; Stiglitz, 2010; Stigler, 1971; Stern, 2003; Tabarquino, 2018; Tenenbaum, 1995; Varela, 2008).

Las investigaciones en materia histórica, jurídica y política de los servicios públicos ${ }^{2}$ en Colombia, esbozan dos periodos: i) Monopolio Estatal desarrollista 1847-1990; y ii) liberalización y mercantilización con criterios de eficiencia y eficacia que consolida el modelo económico neoliberal a partir de 1991 (Camargo, 2015; Cuervo, 2005; Tabarquino, 2018; Varela, 2008).

En continuidad y aporte al ejercicio académico e investigativo, se propone el análisis de la

\footnotetext{
${ }^{2}$ Los servicios públicos se clasifican en domiciliarios y no domiciliarios, el primero hace referencia a los que se articulan de manera directa con el hogar de manera física (agua-alcantarillado, energía, gas en red, telefonía e internet fijo) y el segundo a los que no se relacionan de manera física con el hogar, pero son vitales para el desarrollo humano como salud, educación, seguridad y datos y telefonía móvil.
} 
consolidación legal y regulatoria del sector de las comunicaciones como servicio público, en razón a que los principios de acceso y universalidad que rige el servicio público, son impartidos históricamente por la Unión Internacional de Comunicaciones (UIT), lo cual, implica que el modelo de regulación es de carácter transnacional y las dinámicas organizacionales y empresariales del sector en su consolidación, desarrollo y masificación, son generadas por plataformas mundiales y dinámica en red (Aldana y Vallejo, 2010; Aubin y Verhoest, 2014; Barón y Gómez, 2012; González, 2017; Jordana y Sancho, 2003; Nurul y Verhoest, 2016; Oviedo y Guerra, 2011; Organización para la Cooperación y Desarrollo Económicos [OCDE], 2014; Tabarquino y Verd, 2019; Tabarquino, 2018; Tello, 2015).

\section{Metodología}

Las unidades de análisis para el ejercicio de la investigación, serán las organizaciones e instituciones que participan en la prestación del servicio público en cuestión, dada la dinámica regulatoria (Aubin y Verhoest, 2014; González, 2017; Jordana y Levi, 2005; Nurul y Verhoest, 2016; Tabarquino, 2018): i) Reguladores. Gobierno o agencias gubernamentales que ejercen el marco legal vigente, que define las funciones y responsabilidades en el proceso de toma de decisiones en el sector; ii) Regulados. Agentes privados, públicos y mixtos que realizan la prestación del servicio público; y iii) No regulados. Agentes público-sociales o privados de orden nacional o internacional que representan alguna colectividad, inciden en procesos y actividades que están por fuera de las competencias del marco regulatorio. A continuación, se especifican en la Tabla 1:

\section{Tabla 1}

Empresas, Organizaciones e Instituciones que participan en la consolidación legal y regulatoria del sector de las Comunicaciones en Colombia.

\begin{tabular}{cc}
\hline Actores Reguladores & Actores Regulados \\
\hline Ministerios & Operadores de Servicios de TIC \\
Agencias Nacionales de Regulación & Televisión \\
Comisiones de Regulación & Telefonía Móvil \\
Superintendencias & Internet \\
Autoridades Nacionales & Telefonía Pública Básica conmutada Local \\
Departamentos de Planeación & Contenidos \\
\hline
\end{tabular}




\section{Actores No Regulados}

Organismos de regulación y veeduría internacional

Centros de Pensamiento privados y públicos

Asociaciones Nacionales de Empresas

Asociaciones Nacionales de Usuarios

ONGs y Fundaciones

Universidades

Sindicatos

Partidos Políticos

Fuente: Tabarquino 2018, p. 199.

El enfoque metodológico es mixto (Eisenhardt, 1989; Langley y Royer, 2006; Musca, 2006;

Patton, 2002; Yin, 2003), en donde las técnicas o métodos de análisis de información se centran en:

- Cualitativo. i) Análisis de entrevistas, videos y conferencias en la web; ii) Análisis de entrevistas y conversatorios realizados; iii) análisis documental de revistas, periódicos y estudios sectoriales; iv) Análisis de etnografía retrospectiva: reconstrucción de la evolución del proceso y contexto regulatorio;

- Cuantitativo. i) Análisis de bases de datos de organismos gremiales, institucionales, Públicosociales e internacionales.

Las fuentes de información se enmarcan en: Primarias en cuanto a bases de datos del Ministerio de TIC 2009-2020; y Secundarias; i) Análisis documental de revistas especializadas en TIC, agendas de regulación, leyes de TIC decretos de la Comisión de Regulación de Comunicaciones (CRC), estudios sectoriales de TIC; ii) Entrevistas y video-conferencias en la Web; iii) Informes estadísticos suministrados por el Ministerio de las Tecnologías de las Información y la Comunicación; la Superintendencia de Industria y Comercio (SIC); y la Comisión de Regulación de Comunicaciones-CRC-; Departamento Nacional de Planeación (DNP). 


\section{Resultados y discusión}

\section{Marco legal y regulatorio de inmersión técnica y desarrollo de la conectividad del territorio (1847-1899)}

Este periodo se caracteriza por la incursión del Estado Colombiano en el desarrollo de la infraestructura y la conectividad del territorio mediante el telégrafo eléctrico ${ }^{3}$, que tiene como externalidad negativa la guerra de los mil días entre los años 1899-1902, que trae como consecuencia la destrucción de la infraestructura y avance de las redes de conectividad (Pulido, 2002, p. 66; Rodríguez, 2012, p. 8; Tabarquino, 2018, p. 230).

En materia de regulación, en el año 1869, se nombra a José Florentino Vesga como primer director general de correos y telegrafía; posteriormente en el año 1873, por directrices de seguridad y estrategia militar, la dirección nacional de correos se adscribe a la Secretaría de Guerra y Marina; en el año 1874, se crean las primeras escuelas de telegrafía del país, en los departamentos de Santander, Bogotá y Cauca (Pulido, 2002; Rodríguez, 2012; Tabarquino, 2018).

Los sucesos relevantes en infraestructura y contratación, para el periodo analizado son: i) año 1879, el Estado celebra el contrato con la firma All Américan Cables Inc., con el fin de desarrollar la conectividad mediante cable submarino en el municipio de buenaventura; ii) año de 1880, se concede el permiso de interconexión por medio de cable submarino para consolidar la intercomunicación con Panamá y cualquier Estado de América central mediante la empresa South American Telegraph Co; iii) año 1882, la Ley 21 aprueba el contrato del Estado colombiano con la firma Frulich Murpy \& Co., para establecer el cable submarino telegráfico entre Panamá y Callao, que tendría en su ruta a Buenaventura; y iii) año 1887, la Ley 115 de Junio 28, aprueba el contrato del Estado colombiano con la compañía americana de Cable del

\footnotetext{
${ }^{3}$ La infraestructura y conectividad del telégrafo eléctrico inicia en la presidencia de Tomas Cipriano de Mosquera en el año 1847 y se consolida con la presidencia de José Hilario López en el año 1851, el cual, realiza la contratación de la introducción del Telégrafo y el presidente Manuel Murillo Toro inaugura la primera línea de Bogotá-Nare en el año 1865; posteriormente en el departamento de Antioquia en el año 1886, se inicia la construcción de la línea telegráfica Medellín-Manizales y contratos para el desarrollo de la línea Honda-Manizales (Pulido 2002; Rodríguez 2012).
} 
Pacífico para desplegar el cable submarino del Atlántico; y iv) en mismo año, la Ley 83, autoriza la construcción de las líneas entre Colón y Panamá (Pulido, 2002; Rodríguez, 2012; Tabarquino, 2018).

En lo relacionado con la prestación del servicio: i) El Estado colombiano asume la administración de la empresa de Telégrafos Davinson Wolsey y Stiles en el año 1870, por el no acuerdo del contrato; ii) se inaugura en el año de 1884, el servicio telefónico de carácter privado en Bogotá a cargo de la Empresa Colombiana de Teléfonos; iii) el Estado colombiano autoriza a la firma belga Mourlon and Co. en el año de 1887, para prestar el servicio telefónico de larga distancia entre varias ciudades del país (Cortés, 2012, p. 1; Revista Dinero, 2010, p. 2; Tabarquino, 2018, p. 330); iv) se inaugura en el año 1891, en Medellín el sistema de telefonía pública (Pulido, 2002; Rodríguez, 2012; Tabarquino, 2018); y v) La multinacional Ericcson vende los primeros 50 aparatos telefónicos al Estado Colombiano en el año de 1896 (Tabarquino, 2018).

\section{Marco legal y regulatorio para la recuperación de la infraestructura y el desarrollo de la telegrafía inalámbrica (1900-1942)}

Este periodo se caracteriza por la reconstrucción del telégrafo en el país (Pulido 2002, pp. 4654; Rodríguez 2012, p. 15; Tabarquino 2018, p. 242) ${ }^{4}$; esto inicia con la contratación de Francisco J. Fernández Bello, el 12 de febrero de 1903, por parte del presidente José Manuel Marroquín, posteriormente el proceso es ratificado por el presidente Rafael Reyes (1904-1909), lo cual, logra un avance significativo en el año de 1908 de 16.632 kilómetros de instalaciones telegráficas, con 524 oficinas y 13 líneas. En el año de 1909, la empresa Siemens \& Halske introduce en Colombia los primeros telégrafos de tipo Hugues, que se instalan en Bogotá y Socorro; a partir de 1911, se establecen estaciones inalámbricas por la empresa United Fruit Company, en las ciudades de Santa Marta, Cartagena y San Andrés, con la finalidad de consolidar la comunicación de los barcos entre el Caribe y Estados Unidos (Pulido, 2002; Revista Dinero, 2010; Rodríguez, 2012; Tabarquino, 2018).

\footnotetext{
${ }^{4}$ Después de la guerra de los mil días (1899-1902) quedaron apenas unos 6.000 kilómetros de línea telegráfica, con servicio deficiente (Pulido 2002; Rodríguez 2012; Tabarquino 2018).
} 
En materia de regulación, mediante la Ley 110 (art. 137 y 138) del año 1912, el Estado colombiano, asume de manera directa la prestación de los servicios de correo, telégrafo y comunicaciones, así como la potestad de otorgar permisos a personas naturales o jurídicas para la prestación del servicio de comunicaciones en el territorio; para la consolidación y desarrollo del sector, el mismo año, se contrata por parte del Estado Colombiano a la compañía Gasellschanft fur Drahtlose Telegraphie de Berlín para la construcción de las estaciones en la ciudad de Cartagena y la isla de San Andrés para garantizar la conectividad y comunicación entre Colombia, Nueva York y Cuba (Pulido, 2002; Rodríguez, 2012; Tabarquino, 2018). Se sanciona la Ley 124 del año 1913, la cual, nacionaliza los servicios de correo, crea normas de franquicia y elimina la prestación del servicio público de correo por parte de entidades territoriales Municipios y Departamentos; en el mismo año la empresa Marconi Wireless Telegraph Company, establece la prestación del servicio público inalámbrico en las ciudades de Bogotá, Medellín y Buenaventura; seguidamente en el año de 1915, la All America Cables and Radio conectó a Buenaventura con Ecuador y Panamá (Pulido, 2002; Rodríguez, 2012; Tabarquino, 2018).

La Ley 56 del año 1922, mediante la declaración de la utilidad pública de las empresas prestadoras, permite a los concejos municipales el fomento de los servicios de comunicaciones fuera de los límites jurisdiccionales del municipio; la Ley 31 del año 1923, crea el Ministerio de Correos y Telégrafos para regulación y control del sector, como también la potestad de prestar directamente los servicios postales y la telegrafía en el orden nacional; la Ley 54 del año 1927, autoriza al gobierno la construcción de comunicaciones telefónicas de larga distancia; la Ley 198 del año 1936, otorga al Estado, la potestad de prestar servicios de comunicaciones de manera directa o por medio de concesiones, faculta a los departamentos y municipios en la oferta de servicios telefónicos previa aprobación del Gobierno (Pulido, 2002; Rodríguez, 2012; Tabarquino, 2018) .

\footnotetext{
${ }^{5}$ Cabe señalar que el año de 1932, se crea la Unión Internacional de Telecomunicaciones (UTI) mediante la firma del convenio universal de Telecomunicaciones que implica la Unión de Radiotelegrafía y el Convenio Universal de Telecomunicaciones.
} 
La apuesta al desarrollo de infraestructura de comunicaciones mediante la Ley 76 de 1914 que permite la consolidación de estaciones inalámbricas; la Firma Marconi Wireless Telegraph Co , es contratada por el gobierno colombiano en el año 1919, para la construcción de la Estación Internacional de Bogotá y la estaciones de Barranquilla, Medellín y Cúcuta; a su vez se realiza un contrato con la firma The Central and South American Telegraph en lo relacionado con el cable submarino entre Cartagena y Colón.

La Ley 43 del año de 1921, dinamiza la construcción y operación de redes de larga distancia por parte de los departamentos en sus respectivas jurisdicciones con recursos propios o participación de capital privado (Pulido, 2002; Rodríguez, 2012; Tabarquino, 2018).

En lo relacionado con la prestación del servicio, se establece en el año 1929, la primera empresa interdepartamental en Colombia de comunicaciones con participación de propiedad norteamericana y alianza con la Empresa de Teléfonos de Bogotá, la cual, se denominó Compañía Telefónica Central; en el año de 1884, se crea la Empresa de Teléfonos de Bogotá (ETB); en el año de 1941, se reconoce al Estado colombiano la participación del 4\% del Producto Bruto, por parte de las dos grandes empresas privadas que concentraban la prestación del servicio de comunicaciones en Colombia Marconis Wlreless Telegraph Co y al All America Cables and Radio (Pulido, 2002; Rodríguez, 2012; Tabarquino, 2018).

\section{Marco legal y regulatorio para la consolidación jurídica y nacionalización de las comunicaciones (1943-1989)}

Este periodo se caracteriza por la nacionalización de las comunicaciones en el territorio colombiano, con ideología de unificar los servicios bajo el control estatal colombiano (Pulido, 2002, pp. 55-68; Rodríguez, 2012, p. 18; Tabarquino, 2018, p. 256; Uribe, 2005, pp. 7-8).

En lo relacionado con la Regulación, prestación y operación del servicio, la Ley 6 del año 1943, establece la estrategia de la compra de empresas existentes como Marconi Wireless Telegraph Company; la Ley 83 del año 1945, crea la Empresa Nacional de Radiocomunicaciones; en el año 1947, se compra la empresa interdepartamental Compañía 
Telefónica Central, para lograr la consolidación de una Empresa Nacional de comunicaciones con autonomía administrativa y patrimonio propio; el Decreto 1684 de mayo 23 de 1947, unifica la prestación de servicios telefónicos, radiotelefónicos y radiotelegráficos, absorbiendo las centrales interurbanas de servicio telefónico de larga distancia (Pulido, 2002; Rodríguez, 2012; Tabarquino, 2018). El Decreto 2145 del año 1949, reglamenta la interconexión de redes telefónicas interurbanas; el Decreto 1233 del año 1950, crea TELECOM ${ }^{6}$ (servicios telegráficos inalámbricos y telefónicos a larga distancia), fusionando la empresa nacional de Comunicaciones y la Empresa Nacional de Radiocomunicaciones. En el año 1965, TELECOM hace parte de INTELSA ${ }^{7}$ como accionista (Pulido, 2002; Rodríguez, 2012; Tabarquino, 2018; Uribe, 2005); en el año 1953, el ministerio de Correos y Telégrafos pasa a la denominación de Ministerio de Comunicaciones. En el año 1954 se inaugura la televisión colombiana (Pulido, 2002; Tabarquino, 2018; Uribe, 2005). En el año 1960, la empresa privada All American Cables Inc, retira la prestación del servicio de comunicaciones en el país a raíz que el Decreto 1233 nacionaliza totalmente los servicios telegráficos nacionales e internacionales; se funda el instituto de Electrónica y de Telecomunicaciones (ITEC) en Bogotá en el año 1961; la UTI (Unión Internacional de Comunicaciones) mediante la Ley 26 del año 1974 ratifica el convenio con el gobierno colombiano; en el año 1987 Colombia se convierte en signatario de INMARSAT $^{8}$ donde es miembro hasta la fecha (Pulido, 2002; Tabarquino, 2018; Téllez, 2014; Uribe, 2005).

En materia tarifaria, el Decreto Ley 3069 de 1968, crea la Junta Nacional de Tarifas de Servicios Públicos adscrita al DNP (Departamento Nacional de Planeación), con la finalidad de regular, vigilar y controlar la prestación y cobro de los servicios públicos por parte de las empresas municipales, regionales y departamentales. La fijación de las tarifas, tomaba como base el avalúo catastral, el cual, se sostuvo 15 años en el cálculo de la estructura tarifaría; la Ley 14 de 1983, incorpora en el cálculo de la estructura tarifaría la Estratificación Socioeconómica de

\footnotetext{
6 “TELECOM como prestadora de servicios de telefonía local, dinamizó y posibilitó el sector con aumentos importantes en cobertura. Más o menos en el año de 1992, la empresa había constituido 11 sociedades regionales de telecomunicaciones, conocidas como empresas teleasociadas, que operaban 182 mil líneas. Esta operaba directamente el servicio en 428 cabeceras municipales, con 394 mil líneas" (Arenas, 1992, citado por Uribe, 2005, p. 8, párr. 3).

${ }^{7}$ Consorcio Internacional de Comunicaciones por Satélite creado en el año 1964 (Pulido, 2002, p. 59).

${ }^{8}$ Compañía Internacional con sede en Inglaterra que provee soluciones de Servicios Satelitales Móviles (SSM).
} 
las viviendas, que adiciona variables relacionadas con materiales de construcción, ubicación, vías de acceso y zonas verdes (Pulido, 2002, pp. 55-68; Tabarquino, 2011, p. 17; Tabarquino, 2018, p. 256).

En cuanto a desarrollo de infraestructura y contratación, la empresa de fabricación e innovación de comunicaciones, Ericsson aporta al desarrollo de Colombia, año 1958, instaura la primera central privada PABX en Colombia, año 1974, se protocoliza la ampliación del sistema de larga distancia mediante la facturación automática en Bogotá, año 1977, desarrolla ensamblaje local - fabrica- y en el año de 1980 instaura la primera central AXE fabricada en Colombia (Pulido, 2002; Tabarquino, 2018). Otro actor importante de la época TELECOM, en el año de 1968, contrata los servicios de COMSAT $^{9}$, para desarrollar el proyecto de la utilización de satélites de comunicación para mejorar los servicios internacionales. La Ley 72 del año 1989, establece los principios y objetivos de la prestación del servicio público de telecomunicaciones (Pulido, 2002; Tabarquino, 2018; Uribe, 2005).

\section{Marco legal $y$ regulatorio para la liberalización $y$ mercantilización de las telecomunicaciones (1990-2009)}

Este periodo se caracteriza por una regulación, basada en la liberalización y mercantilización de las comunicaciones en Colombia, inicia con la aprobación y aplicación del Decreto 1900 de 1990 (art. 4), el cual, legitima las bases de la participación privada, la eliminación de monopolios estatales y la clasificación de los servicios públicos de telecomunicaciones (ASOCEL, 2004; CINTEL, 2006, 2007; CRC, 2010; CRT, 2010; Montenegro y Niño, 2001; Revista Dinero, 2010; Tabarquino, 2011; Tabarquino, 2018 ; Uribe, 2005), a continuación se referencia la clasificación de los servicios de telecomunicaciones en la Tabla 2:

${ }^{9}$ Corporación Mundial de Satélites, empresa norteamericana regulada y creada congreso de los Estados Unidos (Pulido, 2002, p. 59). 


\section{Tabla 2}

Clasificación de los Servicios de Telecomunicaciones

\begin{tabular}{|c|c|}
\hline Categoría & Servicio \\
\hline \multirow{4}{*}{ Servicios Básicos } & Telefonía Fija \\
\hline & Telefonía Móvil \\
\hline & Telefonía Móvil - celular \\
\hline & Telegrafía y Telex \\
\hline \multirow{2}{*}{ Servicios de Difusión } & Televisión \\
\hline & Radio \\
\hline \multirow{3}{*}{ Servicios Telemáticos } & Telefax \\
\hline & Videotex \\
\hline & Datafax \\
\hline \multirow{2}{*}{ Servicios de Valor Agregado } & Correo Electrónico \\
\hline & Transferencia Electrónica de Fondos \\
\hline \multirow{2}{*}{ Servicios Auxiliares } & Meteorología \\
\hline & Navegación Aérea o Marítima \\
\hline \multirow{2}{*}{ Servicios Especiales } & Radioaficionados \\
\hline & Investigación \\
\hline
\end{tabular}

Fuente: tomado del Decreto 1900 de 1990.

Posteriormente, el Decreto 2123 de 1992, convierte a TELECOM en empresa industrial y comercial del Estado, dando lugar, al inicio de la privatización y subasta de la empresa pública más grande de telecomunicaciones de Colombia (Tabarquino, 2018).

El marco regulatorio del sector de la Telecomunicaciones en Colombia 1990-2009, se establece a partir de la Ley 142 de 1994 (Ley de Servicios Públicos Domiciliarios) ${ }^{10}$, que además de regular el sector en estudio, también regula sectores como acueducto, alcantarillado, aseo, energía eléctrica, distribución de gas combustible, telefonía fija pública básica conmutada, telefonía local móvil en el sector rural; la Ley inserta y dinamiza el principio de libertad de empresa (artículo 10) y define mediante el artículo 11, la función social que posee la propiedad de las empresas prestadoras de servicios públicos (Duque, 2011, p. 9; Tabarquino, 2018, p. 270; Varela, 2008, p. 4).

\footnotetext{
${ }^{10}$ La Ley 142 de 1991 sobre los servicios públicos domiciliarios, se dictó en cumplimiento de los artículos 365 a 370 de la Constitución de 1991, en los cuales se establece que los servicios públicos pueden prestarse por, particulares y las condiciones que se deben tener para tal efecto, lo cual, no permitía la Constitución de 1886. Los constituyentes del 91, trajeron dichos artículos a la Constitución debido a que el Estado no estaba en condiciones de prestar los nuevos servicios de las comunicaciones que se avizoraba en ese momento que llegarían con las nuevas tecnologías y en especial con la unión de la telefonía celular y las tecnologías informáticas.
} 
La Ley 142 de 1994 define la estructura institucional para el diseño de las políticas en el sector de telecomunicaciones en cuanto a regulación, vigilancia y el control. Las entidades encargadas por la Ley para cumplir estas funciones son: i) Ministerio de Comunicaciones; ii) Comisión de Regulación de Telecomunicaciones (CRT); iii) Colombia de Telecomunicaciones (antes TELECOM); iv) Departamento Nacional de Planeación; v) Superintendencia de Servicios Públicos Domiciliarios (Uribe, 2005, p. 24). Las funciones del Ministerio de las Comunicaciones, se establecen mediante el Decreto 2122 de 1992, que a su vez crea la Comisión de Regulación de Telecomunicaciones (CRT).

El marco regulatorio de las telecomunicaciones en Colombia entre los años 1991-2009, aplicaba el régimen tarifario de la siguiente forma (ASOCEL, 2004, Cortés, 2012; CINTEL, 2006, 2007, 2013; CRC, 2010; CRT, 2010; Fedesarrollo, 2011; Montenegro y Niño, 2001; Tabarquino, 2011; Tabarquino, 2018; Uribe, 2005):

- El servicio público de telefonía pública básica conmutada (TPBCL), es regulado por la Ley 142 de 1994 (Ley de Servicios Públicos domiciliaros); el Ministerio de las Telecomunicaciones y a la Comisión de Regulación de Telecomunicaciones (CRT), ejercían el control tarifario mediante el artículo 126 de la ley, en donde las fórmulas tarifarias contaban con una vigencia de cinco años y que, vencido dicho período, continuarán rigiendo mientras la comisión no fijara unas nuevas o una nueva estructura tarifaria.

- El servicio de Telefonía Móvil Celular, era regulado por la Ley 37 de 1993, la cual, incluye la figura de los contratos de asociación Joint Venture que permite que una organización de naturaleza pública-estatal se pueda asociar con organizaciones privadas para la prestación de servicios de telecomunicaciones, donde se comparte ingresos y riesgos propios del sector (Uribe, 2005, p. 14). 
El modelo de regulación para las telecomunicaciones 1990- 2009, se basaba en el esquema de la Regulación de Precios Máximos $(\mathrm{RPI}-\mathrm{X})^{11}$ y con un régimen de regulación vigilado ${ }^{12}$, a continuación se esboza en la Tabla 3:

\section{Tabla 3}

Modelo de Regulación de las Telecomunicaciones 1990-2009

$\begin{array}{ll}\text { Comisión } & \text { CRT }- \text { Comisión Reguladora de } \\ \text { Reguladora } & \text { Telecomunicaciones }\end{array}$

\begin{tabular}{|c|c|}
\hline $\begin{array}{l}\text { Servicio } \\
\text { Público }\end{array}$ & $\begin{array}{l}\text { TPBC - Telefonía pública básica conmutada: i) TPBCL - Telefonía pública } \\
\text { básica conmutada local; ii) TPBCLE - Telefonía pública básica conmutada local } \\
\text { extendida; iii) TPBCLD - Telefonía pública básica conmutada de larga } \\
\text { distancia; iv) TPBCLDN - Telefonía pública básica conmutada de larga } \\
\text { distancia nacional; v) TPBCLDI - Telefonía pública básica conmutada de larga } \\
\text { distancia internacional; vi) TMR - Telefonía local móvil en el sector rural }\end{array}$ \\
\hline $\begin{array}{l}\text { Régimen de } \\
\text { Regulación }\end{array}$ & Régimen de Libertad Vigilada ${ }^{13}$ \\
\hline $\begin{array}{l}\text { Resoluciones } \\
\text { Marco } \\
\text { Regulatorio }\end{array}$ & $\begin{array}{l}\text { *Resolución } 087 \text { de 1997. "Por medio de la cual se regulan en forma integral los } \\
\text { servicios de Telefonía Pública Básica Conmutada (TPBC) en Colombia". } \\
\text { *Resolución } 575 \text { de } \mathbf{2 0 0 2} \text {. "Por la cual se modifica la numeración de la } \\
\text { Resolución CRT } 087 \text { de } 1997 \text { y se actualizan sus Modificaciones en un solo } \\
\text { cuerpo resolutivo". }\end{array}$ \\
\hline
\end{tabular}

Fuente: Tabarquino (2011, p. 13).

Tabarquino (2011, p. 227-229), señala que el esquema regulatorio de precios máximos, fija un máximo o rango hasta dónde puede llegar la empresa ${ }^{14}$; por lo cual, la demandada superará a la

\footnotetext{
11 "Esta forma de regulación basada en los precios máximos, es un método que se centra directamente en los precios de los propios servicios, es decir, que mediante un control directo de los precios, que incluye la introducción de ajustes para tener en cuenta el efecto inflación, la autoridad reguladora puede garantizar unos precios razonables a los usuarios del servicio, con independencia de los factores que se empleen para conseguirlo" (Mochón, 1995, p. 149).

12 El servicio de Telefonía Pública Básica Conmutada (TPBC)-“Régimen de Libertad Vigilada"-La resolución 087 de 1997.

El servicio público de telefonía celular - "Régimen especial” Al respecto, las resoluciones 087 de 1997 "Los servicios de telefonía móvil (...) estarán sometidos al régimen vigilado de tarifas".

${ }^{13}$ El régimen de libertad vigilada se define como aquel "Régimen de tarifas mediante el cual las empresas de servicios públicos domiciliarios determinan libremente las tarifas de venta a medianos y pequeños consumidores, con la obligación de informar por escrito a las comisiones de regulación sobre las decisiones tomadas sobre esta materia" (Ley 142 de 1994, art. 14).
} 
oferta, los ingresos y las utilidades de la empresa deben tener como parámetro lo que establezca la Comisión de Regulación de Telecomunicaciones.

La metodología RPI- $X^{15}$, se aplica en Colombia de manera similar en los servicios de energía, gas por red, Gas Licuado de Propano (GLP) y aseo; es un modelo que se introduce a partir del informe elaborado por el profesor Littlechild en el año de 1983 para el Departamento de Industria (Regulation of British Telecommunications Profitability). El sistema denominado de precio tope o price cap, se desarrolla en Gran Bretaña, siendo la principal modalidad práctica de regulación por incentivos.

Este sistema es representado por la fórmula RPI-X y basado en el control de precios de los servicios básicos, bajo la presunción de que si el precio no puede aumentarse. La única fórmula para elevar los beneficios es reducir los costos, es decir, que el sistema se basa en limitar el incremento normal del precio, de un conjunto de servicios básicos en el porcentaje que resulta de reducir el IPC (en Gran Bretaña, Retail Price Index o RPI), en función de un factor X que refleja lo mejor de la eficiencia de la empresa y es determinado por el ente regulador ${ }^{16}$.

${ }^{14}$ La política pública de regulación es de carácter técnico sustentado en el concepto de precio que, a la manera de ver la receta del Consenso de Washington, aplica la medida económica de la inflación como parámetro central de la economía.

${ }^{15}$ Resoluciones de la CRT Nº 087 de 1997 y N 575 de 2002

*El Cálculo y ajuste del Costo Máximo (CM), CM t =CM t-1 (1 + IPC - X), Donde: CM: Costo Máximo permitido por la CRT para el plan tarifario básico.; IPC: Meta del incremento anual del índice precios al consumidor proyectado por el Banco de la República para el año t; X: Factor de productividad, correspondiente al dos por ciento $(2 \%), \mathbf{t}$ : Corresponde al año de aplicación.

*Internet por Medio de la Red de Telefonía Pública Básica Conmutada Local (TPBCL), Tt = Tt-1 (1 + IPC - X)*Q , Donde: T: Tarifas para las llamadas de TPBCL cuando se accede a Internet; IPC: Meta del incremento anual del Índice Precios al Consumidor proyectada por el Banco de la República para el año t; X: Factor de productividad, correspondiente al dos por ciento (2\%); $\mathbf{t}$ : Corresponde al año de aplicación; Q: Factor de calidad

16 Este sistema se aplica en el Reino Unido en los años 80 por que el gobierno Thatcher toma la decisión de privatizar las empresas de servicios públicos y desarrollar un marco institucional que permitiera alinear los incentivos del sector privado con las necesidades de los usuarios "Durante los 80, en Inglaterra se transfirieron al capital privado las empresas de telecomunicaciones, energía y gas, y en el sector de acueductos se desarrolló un marco que permite la entrada de capital privado. En pocos años, el nuevo marco mostró sus bondades en términos de eficiencia productiva y expansión de los sistemas. Alrededor de este proceso, se generó un acervo de teoría económica que establece los mecanismos para controlar el poder monopólico y oligopólico característico de estos servicios y brinda pautas para el diseño de mercados eficientes en industrias de redes. De igual forma, se desarrollaron los parámetros para implementar una estructura institucional de regulación y control para buscar que los resultados de la prestación de los servicios se asemejen a los de un mercado competitivo" (Roda, 2004). 
En relación a la prestación del servicio y operatividad del sector, en el año 1994, el Estado colombiano, abre la licitación de la operación de telefonía celular, adjudicando licencias y contratos de concesión a las compañías Comcel (hoy Claro) y Celumóvil (posteriormente adquirida por Bell South y luego por Telefónica, hoy Movistar). En 2003, ETB y EPM crearon Colombia Móvil, que con su marca Ola se convertiría en el tercer jugador del mercado. En el 2006, Millicom compró la mayoría accionaria de la empresa y cambió su marca a Tigo (Revista Dinero, 2010; Tabarquino, 2018).

En cuanto a la telefonía pública básica conmutada de larga distancia nacional e internacional, el Estado colombiano, mediante la resolución 28 de 1995 (art. 4,5 y 6), autoriza a los tres operadores para prestar el servicio: Orbitel, ETB y Telecom. Orbitel mantuvo acuerdos de conexión de redes y operatividad con las compañías de celulares Cocelco, Comcel, Celcaribe, Occel, Celumovil-Bellsout; y además, dentro de los accionistas de Orbitel (EPM, Organización Carlos Sarmiento Angulo y el Grupo empresarial Bavaria) (Pulido, 2002, p. 183-189).

En cuanto a desarrollo de infraestructura y contratación, la empresa de fabricación e innovación de comunicaciones Ericsson, aporta al desarrollo de Colombia, en el año 1994, porque, dinamiza las primeras redes de celulares AMPS (El Sistema Telefónico Móvil Avanzado), en el año 2001 opera la primera red GSM (sistema global para las comunicaciones móviles), posteriormente en el año 2003 entra en funcionamiento con la primera red Móvil CDMA (acceso múltiple por división de código); y en el año 2005 maneja la primera red de cobertura rural GSM. En los años siguientes, es el principal suministrador de banda ancha fija (año 2005), introduce la banda ancha móvil (3G/WCDMA) y en el año 2009, opera el Sistema Internet Protocolo Televisión (IPTV) (Tabarquino, 2018).

Otro actor importante en el desarrollo de infraestructura y contratación para este periodo de análisis es ANDITEL, un operador de soluciones de telecomunicaciones y tecnologías digitales que tiene como finalidad aplicar integración para proveer conectividad, construir y gestionar soluciones y plataformas especializadas; ha participado y contratado con el gobierno de manera directa y por licitación en proyectos como estaciones satelitales (año 1999), sistemas telefonía 
rural - sistema multiacceso (año 2000), Compartel (año 2004 y 2005), Conexión Total (año 2007 y 2008).

El Gobierno Nacional, en la perspectiva de un mejor posicionamiento en la economía global, aumento el bienestar social y disminución de la brecha digital; realizó una serie de apuestas de política pública e inversiones asociadas a las telecomunicaciones en la última década:

- El Plan Nacional de Servicio Universal 1999-2009 (PNSU), define el alcance y la función social de las telecomunicaciones en Colombia, marcando el derrotero para la creación y consolidación de los principales programas y planes de inversión en TIC.

La propuesta "Visión Colombia II Centenario: 2019", plantea lineamientos estratégicos para el desarrollo del país, donde las TIC son la base para una infraestructura adecuada para el desarrollo y una sociedad informada.

Los documentos CONPES 3063 de 1999 "Programa de donación masiva de computadores a colegios públicos - Computadores para Educar", 3072 de 2000 "Agenda de Conectividad - un salto a Internet" y 3171 de 2002 "Lineamientos de política de telecomunicaciones sociales 2002-2003", dieron origen a: los programas Computadores para Educar, Gobierno en Línea y Compartel.

Existen casos de éxito a nivel de proyectos piloto, que se han llevado a cabo en Colombia, en los que se ha evidenciado que las Telecomunicaciones son herramientas contundentes en la consolidación de actividades productivas, provisión y desarrollo en las zonas territoriales de menores ingresos (Telefonía Social -1997-, Compartel -1999-, Computadores para Educar 2000- y la Agenda de Conectividad -2000-).

\section{Marco legal y regulatorio para la consolidación de la convergencia tecnológica (2009-2020)}

En el año 2009, se delimita el marco regulatorio de Las TIC en Colombia mediante la Ley 1341 - Ley de TIC o Ley de Convergencia- que define: i) los principios y conceptos de sociedad de la información y organización de TIC, en relación a la apertura de la competencia internacional, la inclusión y el desarrollo del concepto de ciudadanía digital y la base para el entendimiento del 
concepto de nativo digital; ii) crea la Agencia Nacional del Espectro como ente regulador y estratégico en la sector de las TIC, sustentado en la importancia de la conectividad y en el juego de nuevos actores que compiten en un orden de infraestructura, servicios y aplicaciones; e iii) integra el sector de la informática y sector de telecomunicaciones como sector de las TIC (Tecnologías de la Información y las Comunicaciones) (Aldana y Vallejo, 2010; Barón y Gómez, 2012; CRC, 2010; Tabarquino, 2012, 2013; Tabarquino, 2018).

La Ley 1341 del 2009, se compone de 73 artículos que determinan y regulan: i) competencia de redes y mercados; ii) la política pública del espectro radioeléctrico; iii) legitima la competencia e incentiva la inversión en el sector; iv) mayor cobertura, acceso y penetración de servicios de banda ancha (Aldana y Vallejo, 2010, p. 175; Tabarquino, 2018, p. 292).

La Ley define en su artículo 6 que las TIC, son un "conjunto de recursos, herramientas, equipos, programas informáticos, aplicaciones, redes y medios, cuya finalidad es la compilación, procesamiento, almacenamiento, transmisión de información (voz, datos, texto, video e imágenes)". El artículo 9, establece la composición del sector de las TIC en Colombia por "industrias manufactureras, comerciales y de servicios, cuyos productos recogen, procesan, crean, transmiten o muestran datos e información electrónicamente".

El artículo 2, de la ley, define ocho principios: i) acceso y uso de las TIC ; ii) Libre competencia; iii) eficiencia de la infraestructura y de los recursos escasos; iv) Protección a los derechos de los usuarios; v) Promoción de la Inversión; vi) Neutralidad Tecnológica; vii) El Derecho a la comunicación, la información y la educación y los servicios básicos de las TIC; viii) Masificación del gobierno en línea (Aldana y Vallejo, 2010; CRC, 2010; OCDE, 2014; Oviedo y Guerra, 2011; Tabarquino, 2012, 2013; Tabarquino, 2018).

El artículo 23, establece que los proveedores de redes y servicios de TIC, podrán fijar libremente los precios al usuario, es decir que el mercado se encuentra en un régimen de libertad y además la Comisión de Regulación de Comunicaciones interviene regulando precios cuando no exista competencia, se presente una falla de mercado o cuando la calidad de los servicios ofrecidos no 
se ajuste a los niveles exigidos. La Comisión de Regulación de Comunicaciones (CRC), hace énfasis en la regulación de mercados mayoristas ${ }^{17}$.

La Ley 1341 de 2009, consolida y desarrolla un nuevo esquema regulatorio, la convergencia tecnológica $^{18}$, que exige que las redes sean multiservicios e implica una regulación por mercados, que es la posibilidad de ofrecer a los usuarios en un mismo dispositivo aplicaciones de Voz, datos, audio y video. Lo anterior propicia al desarrollo complejo del derecho y la política pública en los mercados tecnológicos, es decir, que el nuevo esquema regulatorio entra en la dinámica de nuevos servicios generados partir de los avances tecnológicos, la liberalización en la prestación de servicios, la no existencia de monopolio público, una complejidad más alta en las actividades y relaciones de los proveedores de sistemas, infraestructura y equipos acompañada de estímulos a la innovación y a la inversión por parte del Estado y la empresa privada (Aldana y Vallejo, 2010; CRC, 2010; Duque, 2011; OCDE, 2014; Oviedo y Guerra, 2011; Tabarquino, 2012, 2013; Tabarquino, 2018) ${ }^{19}$

En Colombia las instituciones que finalmente consolidan e implementa, la política pública de regulación de las TIC, entre el periodo 2009-2020, son principalmente: El MinTIC, la

\footnotetext{
17 "Los mercados mayoristas son estructuras físicas donde se realizan intercambios comerciales entre profesionales, reunidos con el objetivo de comparar y vender productos...se observa también ...la presencia de diferentes tipos de operadores de servicios logísticos, financieros y de otras actividades" (Green, 2003).

18 "Posibilidad de ofrecer a los usuarios en un mismo dispositivo aplicaciones de voz, datos, audio y video" (El Espectador, 29 Jun. 2008)..."Evolución coordinada de redes que antes eran independientes hacia una uniformidad que permita el soporte común de servicios y aplicaciones" (UIT, 2004)..."Es una amplia gama de contenidos (audio, video, texto e imágenes) y servicios se distribuyen a través de distintas redes (fija, de banda ancha, infraestructura móvil, satélite, cable terrestre) a una variedad de dispositivos de consumo (PC, TV, teléfonos móviles)" (OFCOM, $2008,61) \ldots .$. "Está determinada por la combinación de tendencias tecnológicas, financieras y estratégicas que pueden ser independientes de las tecnologías subyacentes" (Henten et al., 2003, p. 33)..."la convergencia de TIC, es la combinación de tendencias tecnológicas, financieras y estratégicas que pueden ser independientes de las tecnologías subyacentes" (Henten, 2003, p. 33, citado por Aldana y Vallejo, 2010, p. 173).

${ }^{19}$ El Gobierno Nacional, en la perspectiva de un mejor posicionamiento en la economía global, aumento del bienestar social y disminución de la brecha digital, realizó una serie de apuestas de política pública e inversiones asociadas en materia de TIC en este periodo regulatorio (Tabarquino 2018): A. Plan Nacional de TIC 2008- 2019 (PNTIC). En este plan, el Gobierno colombiano se compromete a la transversalización del uso eficiente y productivo de las TIC (Duque, 2011; Tabarquino, 2018), para la inclusión social y la competitividad; desarrollando cuatro ejes verticales (Educación, Salud, Justicia, y Competitividad Empresarial) y cuatro transversales (Comunidad, Marco regulatorio, Investigación, Desarrollo e Innovación y Gobierno en Línea). B. Plan Vive Digital 2010-2014; 2014-2018; 2018-2022. El plan, tiene como finalidad masificar los principios orientadores de la "Prioridad al acceso y uso de las Tecnologías de la Información y las Comunicaciones" que establece la ley 1341 de 2009. Este principio, afianza e impulsa la masificación del uso Internet, para reducir la pobreza y generar empleo.
} 
Superintendencia de Industria y Comercio (SIC), la Agencia Nacional del Espectro (ANE), la Agencia Nacional de Televisión (ANTV), la Comisión de Regulación de Comunicaciones $(\mathrm{CRC})^{20}$ y el Departamento Nacional de Planeación (DNP) ${ }^{21}$.

En materia de prestación del servicio los principales operadores de TIC en Colombia son Claro, Movistar, Tigo-UNE, Avantel, Uff Móvil, ETB, Virgin Mobilie y Móvil Éxito (CRC, 2017; Tabarquino, 2018, 2019).

En cuanto a desarrollo de infraestructura y contratación, la empresa Ericsson en el año 2009, opera el Sistema Internet Protocolo Televisión (IPTV), en el año 2012 instaura la primera red 4G/LTE y en el año 2016 el primer servicio VolTE ${ }^{22}$ basado en la Nube (Tabarquino, 2018).

En relación a nuevas innovaciones, surgen los servicios over-the-top $(\mathrm{OTT})^{23}$ o servicios de comunicación audiovisual a través de Internet definidos como video, audio, datos, voz y herramientas de búsqueda que se transmite a través de plataformas de internet móvil o fijo (CRC, 2017; Estavillo, 2014; Lopasso, 2011; Tabarquino, 2018). En Colombia, la cadena de valor de los servicios de comunicación audiovisual (OTT) se compone de los proveedores de servicios, que se describen en la Tabla 4:

${ }^{20}$ La CRC es Unidad Administrativa Especial adscrita al MinTIC, establece el régimen de regulación que incremente el bienestar de los usuarios.

${ }^{21}$ El DNP aporta al sector de las TIC por medio del Plan "Visión Colombia 2019: Avanzar hacia una sociedad mejor informada" que bajo el cumplimiento de diferentes metas se pretende difundir las TIC en los diferentes ámbitos de la sociedad procurando una mayor participación ciudadana con Gobierno en Línea, masificar la información, garantizar el acceso universal a los diferentes servicios de las comunicaciones, en otras metas.

${ }^{22}$ Hacer y recibir llamadas en Alta Definición a través de la red 4G LTE.

23 "Los servicios OTT se han convertido en una preocupación para los operadores de telecomunicaciones cuando empezaron a competir directamente con los servicios ofrecidos tradicionalmente por las compañías de telecomunicaciones: voz, mensajería y pago por TV (pay TV). Por ejemplo, Skype, que ahora pertenece a Microsoft, fue creada en 2003 y en la actualidad cuenta con 250 millones de usuarios activos por mes, quienes hablan 100 minutos en promedio, evitando así el uso de la telefonía tradicional. Tal como Skype se convirtió en un competidor de telecomunicaciones en voz...Finalmente, los servicios OTT son controlados a menudo por compañías poderosas, como los gigantes de la Internet (p. ej. Google, Facebook, Microsoft), los fabricantes de productos electrónicos de consumo (p. ej. Apple, RIM) e incluso las empresas de medios de comunicación (p. ej. Sony Music, Universal), que aseguran el alcance global y la solidez financiera" (Lopasso, 2011, p. 2). 


\section{Tabla 4}

Proveedores de servicios OTT en Colombia

\begin{tabular}{ll}
\hline \multicolumn{1}{c}{ Servicio } & \multicolumn{1}{c}{ Proveedor } \\
\hline Voz & Skype, Facetime, Viper, Kakao, Fring. \\
\hline Mensajería & WhatsApp, Kakao, Facebook, Line Messenger, kik Messenger, \\
& Blackberry Messenger. \\
\hline $\begin{array}{l}\text { TV/Video (Contenidos } \\
\text { audiovisuales) }\end{array}$ & $\begin{array}{l}\text { Netflix, Claro TV, Youtube, Apple Tv, Amazon Video, Movistar } \\
\text { Video, Veo, Totalmovie, Tunes, Vudu, Klic, Crackle. }\end{array}$ \\
\hline Redes Sociales & Facebook, Twitter, LinkedIn, Waze. \\
\hline Herramientas de Búsqueda & Google, el correo web de Microsoft Hotmail. \\
\hline
\end{tabular}

Fuente: elaboración propia, 2020.

En materia de Desarrollo Digital, se consolida de manera legal y regulatoria el Big Data, Economía Digital, la inteligencia artificial, desarrollo espacial, la organización del espectro y ciberseguridad mediante: i) CONPES 3920 del 17 de abril de 2018 -Política Nacional de Explotación de Datos (Big Data)-, tiene como finalidad aprovechar los datos generados por entidades públicas a través de la digitalización e interoperabilidad; iii) CONPES 3975 del 8 de Noviembre de 2019- Política Nacional de transformación Digital e Inteligencia Artificial-, tiene como finalidad la generación de valor económico y social, de datos satelitales, ciberseguridad y subasta del espectro por medio de las TICs; iii) CONPES 3983 del 13 de Enero del 2020 Política de Desarrollo Espacial: Condiciones para el impulso de la competitividad nacional-, la finalidad es la regulación en el largo plazo de bienes y servicios satelitales mediante la consolidación en innovación, emprendimiento y conocimiento tecnológico; y iv) CONPES 3995 del 1 de julio de 2020 -Política Nacional de Confianza y Seguridad Digital-, la finalidad es consolidar las capacidades en seguridad digital para la ciudadanía, el sector público y el sector privado mediante nuevas tecnologías, con principios de inclusión y competitividad digital.

\section{Conclusiones}

El desarrollo regulatorio del sector de las comunicaciones en Colombia entre los años 1847 y 2020, evidencian diferentes ideologías: i) Estado de base conservadora, orgánico, corporativo y de estrecha relación con la religión católica (1847-1942); ii) Estado proteccionista, de bienestar y Regulador, con la aplicación estructural del modelo económico de crecimiento endógeno (1943-1989); y iii) Estado Mercantilista, con la aplicación estructural del modelo 
económico de crecimiento exógeno que marca el derrotero de la implementación del modelo neoliberal con el apoyo de organismos internacionales OCDE, FMI, BM, OMC (1990-2020).

La prestación del servicio público de las comunicaciones en Colombia entre los años 1847-2020, refleja diferentes ejercicios de toma decisiones desde los legal y lo regulatorio: i) entre los años 1847-1889, la prioridad es la inversión privada y contratación internacional para la construcción de infraestructura con un aprendizaje institucional basado en la transferencia de conocimiento técnico y de prestación del servicio; ii) entre los años 1900-1942, las alianzas público-privada en los territorios, consolidan la Institucionalidad en materia de prestación del servicio público en el territorio; iii) entre los años 1943-1989, el ejercicio regulatorio y legal es la nacionalización del sector de las comunicaciones mediante la figura del monopolio estatal; iv) entre los años 19902009, entra en auge la aplicación de las directrices del Consenso de Washington, lo cual, afianza la liberalización y mercantilización del sector de las comunicaciones, con parámetros de libre competencia, desregulación y privatización; y v) entre los años 2009-2020, se establece un marco regulatorio que Integran telecomunicaciones e informática, mediante el concepto de Tecnologías de la Información y las Comunicaciones, para darle paso a la consolidación de la Economía Digital, Ciudades y territorios Inteligentes, Big Data, Internet de las Cosas, Datos en la Nube, etc.

El sector de las comunicaciones en Colombia, tiene una transición de servicio público domiciliario (1847-1989) a servicio público no domiciliario (1990-2020), sustentando en el desarrollo de la convergencia tecnológica y la consolidación de la política internacional de la sociedad de la información y el conocimiento, que promueven dinámicas organizacionales para la consolidación, desarrollo y masificación de plataformas mundiales de universalidad y acceso, en un ejercicio ideológico transnacional y de transferencias de políticas públicas.

\section{Referencias}

(1) Aubin, D. y Verhoest, K. (2014). Multilevel Regulation in Telecommunications: Adaptive Regulatory Arrangements in Belgium, Ireland, the Netherlands, and Switzerland. Palgrave Macmillan Ltd, Basingstoke. 
(2) Aldana, A. y Vallejo, A. (2010). Telecomunicaciones, Convergencia y Regulación. Revista $\begin{array}{llll}\text { de Economía } & \text { Institucional. }\end{array}$ https://revistas.uexternado.edu.co/index.php/ecoins/article/view/2504

(3) Asociación de la Industria Celular en Colombia- ASOCEL- (2004). El Derecho de La Competencia en El Sector de Las Telecomunicaciones.

(4) Barón, L. F. \& Gómez, R. (2012). De la infraestructura a la apropiación social: panorama sobre las políticas de las tecnologías de información y comunicación (TIC) en Colombia. Signo $y$ pensamiento, 31(61), $38-55$. https://revistas.javeriana.edu.co/index.php/signoypensamiento/article/view/4408

(5) Camargo, S. R. M. (2015). Los servicios públicos domiciliarios en Colombia: su prestación, regulación y control. Revista del CLAD Reforma y democracia, (63), 163-194. https://www.redalyc.org/pdf/3575/357542721006.pdf

(6) Consejo Nacional de Política Económica y Social -CONPES 3995-. (2020). Política Nacional de Confianza y Seguridad Digital- Departamento Nacional de Planeación. https://colaboracion.dnp.gov.co/CDT/Conpes/Econ\%C3\%B3micos/3995.pdf

(7) Consejo Nacional de Política Económica y Social -CONPES 3983-. (2020). Política de Desarrollo Espacial: Condiciones Habilitantes Para El Impulso de La Competitividad Nacional. Departamento Nacional de Planeación. https://colaboracion.dnp.gov.co/CDT/Conpes/Econ\%C3\%B3micos/3983.pdf

(8) Consejo Nacional de Política Económica y Social -CONPES 3975. (2019). Política Nacional Para La Transformación Digital e Inteligencia Artificial. Departamento Nacional de Planeación. https://www.mintic.gov.co/portal/604/articles-107147_recurso_1.pdf

(9) Consejo Nacional de Política Económica y Social -CONPES 3920-. (2018). Política Nacional de Explotación de Datos (Big Data). Departamento Nacional de Planeación. https://colaboracion.dnp.gov.co/CDT/Conpes/Econ\%c3\%b3micos/3920.pdf

(10) Cortés, C. J. (2012). Presente y Futuro de las comunicaciones ópticas en Colombia. Primer Seminario Taller Latinoamericano de Instrumentación, Control y Telecomunicaciones SICOTEL 2012. Universidad del Quindío. https://www.academia.edu/6150230/Presente_y_Futuro_de_las_comunicaciones_\%C3\%B3 pticas_en_Colombia_abril_2012

(11)Cuervo, L. M. (2005). Servicios públicos domiciliarios en Colombia: la reforma diez años 
después. Territorios, (14), 27-55. https://www.redalyc.org/pdf/357/35711623003.pdf

(12) Centro de Investigación de las Telecomunicaciones -CINTEL-. (2013). Transformaciones y retos del sector de las TIC en Colombia. https://cintel.co/wpcontent/uploads/2013/05/09_Transformaciones_y_retos_del_sector_TIC.pdf

(13) Centro de Investigación de las Telecomunicaciones -CINTEL-. (2007). Panorama de las Telecomunicaciones en Colombia. https://cintel.co/wpcontent/uploads/2013/05/17.tv_2008_Televisi\%C3\%B3n-abierta-y-porsuscripci\%C3\%B3n.pdf

(14)Centro de Investigación de las Telecomunicaciones -CINTEL-. (2006). Estudio del Sector de las Telecomunicaciones en Colombia. https://cintel.co/wpcontent/uploads/2013/05/23.estudio_sectorial_2006_Estudio-del-Sector-de-la-

Telecomunicaciones-en-Colombia-2006.pdf

(15) Comisión de Regulación de Comunicaciones- CRC- (2017). Hoja de Ruta Regulatoria para el Desarrollo de la Economía Digital en Colombia. https://www.crcom.gov.co/es/pagina/hoja-ruta-economia-digital

(16) Comisión de Regulación de Comunicaciones -CRC- (2010). Análisis del Sector de TIC en Colombia: Evolución y Desafios. https://www.crcom.gov.co/en/pagina/biblioteca-virtual

(17)Comisión de Regulación de Telecomunicaciones -CRT- (2010). El Sector de Las Telecomunicaciones En Colombia 1998-2001. https://www.crcom.gov.co/en/pagina/biblioteca-virtual

(18) Duque, A. C. (2011). La Política Pública de Regulación del Sector de las TIC en Colombia. ¿Servicio Público Domiciliario o Mercancía? http://idoa.consejodeestado.gov.co/documentos/biblioteca/revistas/edi00/doc/art4.pdf

(19)Eisenhardt, M. (1989). Building Theories from Case Study Research. Academy of Management Review, 14(4), 532-550

(20)Estavillo, M. E. (2014). Los Servicios OTT: provisión de contenidos vs televisión abierta y de paga. Instituto Federal de Telecomunicaciones (IFT).

(21) Fedesarrollo. (2011). Impacto de las Tecnologías de la Información y las Comunicaciones (T IC) en el Desarrollo y la Competitividad del País.

(22) González, C. (2017). Measuring and comparing the distribution of decision-making power in regulatory arrangements of the telecommunication sector in Latin America. Journal Utilities 
Policy, 49, 145-155. https://ideas.repec.org/a/eee/juipol/v49y2017icp145-155.html

(23)González, G. (2008). Regulación por Mercados. El Espectador. https://www.elespectador.com/opinion/la-regulacion-por-mercados-columna-23012/

(24) Green, R. (2003). Mercados mayoristas: ¿el inicio de una nueva era? Distribución y consumo, 13(72), 23-37. https://dialnet.unirioja.es/servlet/articulo?codigo=760728

(25) Hiriart, Y., Martimort, D. y Pouyet, J. (2004). On the optimal use of ex ante regulation and $\begin{array}{llll}\text { ex post liability. Economics } & \text { Letters, } & \text { 84(2), 231-235. }\end{array}$ https://www.sciencedirect.com/science/article/pii/S0165176504000862

(26) Jordana, J. y Levi, D. (2005). ¿Hacia Un Estado Regulador Latinoamericano? La difusión de agencias reguladoras autónomas por países y sectores. CIDOB ediciones.

(27) Jordana, J. y Sancho, D. (2003). Policy Networks and The Opening up of the Market: The Case of Telecommunications Liberalisation in Spain. Universitat Pompeu Fabra, Barcelona

(28) Langley, A. \& Royer, I. (2006). Perspectives on Doing Case Study Research in Organizations.M@n@gement, 9(3), 81-94.

(29) Lopasso, G. (2011). Como lidiar con los servicios Over-The-top. Slideshare a Scribd company. https://es.slideshare.net/EricssonLatinAmerica/ott-es

(30) Montenegro, S. \& Niño, L. C. (2001). La Tecnología de la Información y de las Comunicaciones en Colombia. Centro de Estudios de Desarrollo Económico Facultad de Economía Universidad de los Andes. Proyecto Andino de Competitividad. Banco de Desarrollo de América Latina (CAF).

(31) Mochón, F. (1995). Principios de Economía. Madrid: McGraw Hill.

(32) Musca, G. (2006). Une strategie de recherche processuelle: l'étude longitudinale de cas enchâsesés.M@n@gement, 9(3),153-176.

(33) Nurul, A. \& Verhoest, K. (2016). De-facto coordination in regulatory decision-making on liberalized telecommunications sector in Bangladesh and Nepal: a social network analysis. ECPR Standing Group on Regulatory Governance Sixth Biennial Conference Between Collaboration and Contestation: Regulatory Governance in a Turbulent World. RegGov2016, Tilburg University.

(34) Organización para la Cooperación y Desarrollo Económico -OCDE-. (2014). Estudio de la OCDE sobre Políticas y Regulación de Telecomunicaciones en Colombia. 
https://www.oecd-ilibrary.org/science-and-technology/estudio-de-la-ocde-sobre-politicas-yregulacion-de-telecomunicaciones-en-colombia_9789264209558-es

(35) Office of Communications -OFCOM- (2008). Communication market report, London. https://www.ofcom.org.uk/_data/assets/pdf_file/0021/6159/annrep0809full.pdf

(36) Oviedo, J. D. \& Guerra, D. R. (2011). De las telecomunicaciones a las TIC: Ley de TIC de Colombia (L1341). https://www.cepal.org/es/publicaciones/4818-telecomunicaciones-ticley-tic-colombia-11341

(37) Patton, M. Q. (2002). Qualitative evaluation \& research methods (3nd ed.). Thousand Oaks: Sage Publications.

(38) Pulido, I. D. (2002). Evolución del Marco Jurídico de las Telecomunicaciones. [Tesis de Grado, Universidad Javeriana]. Repositorio Institucional - Pontificia Universidad Javeriana http://www.javeriana.edu.co/biblos/tesis/derecho/dere5/TESIS69.pdf

(39) Roda, P. (2004). Impacto sectorial de los diez años de la legislación marco de servicios públicos domiciliarios en Colombia. Estudio Técnico Económica Consultores, Superintendente de Servicios Públicos Domiciliarios.

(40)Rodríguez, J. C. (2012). La Telegrafía: Una revolución en las telecomunicaciones de Colombia: 1865-1923. Banco de la Republica. Bogotá: Credencial. https://www.banrepcultural.org/biblioteca-virtual/credencial-historia/numero-265/latelegrafia-una-revolucion-en-las-telecomunicaciones-de-colombia

(41) Rodríguez, V. (2013). Fallas de Mercado y Regulación Económica: ¿La Regulación ejercida por el Gobierno permite logar un mejor funcionamiento de los mercados? Revista Quipukamayoc. 21(39) 99-111. https://revistasinvestigacion.unmsm.edu.pe/index.php/quipu/article/view/6800

(42)Rozas, P. y Hantke, M. (2013). Gestión pública y Servicios públicos- Notas sobre el concepto servicio público. CEPAL - Serie Recursos Naturales e Infraestructura, 2013. $N^{\circ}$ 162. https://repositorio.cepal.org/handle/11362/6366

(43) Revista Dinero. (2010). Del telégrafo a la convergencia. https://www.semana.com/deltelegrafo-convergencia/95103/

(44) Spiller, P. y Abdala, M. (2000). Institutions, Contracts and Regulation in Argentina. Temas. Buenos Aires. https://www.tandfonline.com/doi/pdf/10.1080/15140326.2001.12040564 
(45) Stiglitz, J. (2010). Regulación y Fallas. Revista de Economía Institucional, 2010. 12(23), 13 28. https://revistas.uexternado.edu.co/index.php/ecoins/article/view/2495

(46) Stigler, G. (1971). The Theory of Economic Regulation The Bell. Journal of Economics and Management Science. 2(1),321. http://pirate.shu.edu/ rotthoku/Liberty/stigler_theory\%20of\%20econ\%20regulation.pdf

(47) Stern, J. (2003). Regulation and contracts for utility services: Substitutes or complements? Lessons from UK Railway and Electricity History. Journal of Policy Reform, 6(4), 193-215. https://www.tandfonline.com/doi/abs/10.1080/134841280410001698978

(48) Tello, D. C. V. (2015). Implementación de tecnologías de la información y las comunicaciones (TIC) en Colombia. Revista de Derecho, Comunicaciones y Nuevas Tecnologías, (14). https://dialnet.unirioja.es/servlet/articulo?codigo=7496889

(49) Tabarquino, R. A. \& Miquel, J. (2019). La tipología de las redes de política pública de regulación del Servicio Público de Comunicaciones en Colombia 1847-2018. Redes, 30(2). https://revistes.uab.cat/redes/article/view/v30-n2-tabarquino-verd

(50) Tabarquino, R. A. (2018). La Red de política pública de regulación del servicio público de las tecnologías de la información y las comunicaciones (TIC) en Colombia 2009-2016: El Acceso (Universalidad) y La Equidad como Principios Rectores. [Tesis Doctorado en Administración. Universidad del Valle].

(51) Tabarquino, R. A. (2018). El servicio público de las Tecnologías de la Información y las Comunicaciones (TIC) en Colombia 2010-2018. Entramado, 14(2), 166-181. https://revistas.unilibre.edu.co/index.php/entramado/article/view/4752

(52) Tabarquino, R. A. (2011). Los Servicios Públicos Domiciliarios en Colombia: Una mirada desde la Ciencia de la Política pública y la Regulación. Eumed.net https://www.eumed.net/libros-gratis/2011c/997/index.htm

(53) Tenenbaum, B. (1995). The Real World of Power Sector Regulation. Viewpoint. No. 50. Washington: Banco Mundial. http://regulationbodyofknowledge.org/wpcontent/uploads/2013/03/Tenenbaum_The_Real_World.pdf

(54) Téllez, L. (2014). Satélites De Telecomunicaciones En Colombia. Pasado Presente y Futuro. Tesis de Grado de Derecho. Universidad De Los Andes.

(55) Uribe, E. (2005). Evolución del Servicio de Telecomunicaciones Durante la Última Década. Bogotá D.C.: Centro de Estudios sobre Desarrollo Económico - CEDE, Universidad de los 
Andes. Colombia.

(56) Universidad Externado de Colombia y UNE - TIGO. (2015). Foro: Regulación de los servicios Over the Top (OTT).

(57) UIT -Unión Internacional de Telecomunicaciones-. (2004). Principios y requisitos para la convergencia de sistemas fijos e IMT-2000 existentes, Recomendación UIT-T Q.1761, http://www.itu.int/rec/T-REC-Q.1761/es

(58)Tello, D. C. V. (2015). Implementación de tecnologías de la información y las comunicaciones (TIC) en Colombia. Revista de Derecho, Comunicaciones y Nuevas Tecnologías, (14), 5. https://dialnet.unirioja.es/servlet/articulo?codigo=7496889

(59) Varela, E. (2008). Las Transformaciones de la Burocracia Pública en Colombia - El impacto de las Políticas neoliberales en el sector de Servicios Públicos Domiciliarios. [Tesis del Doctorado en Administración (Management)] Universidad de Montreal. Escuela de Altos Estudios Comerciales.

(60) Yin, R. (2003). Case Study Research (Third Edition ed. Vol. 5). USA: Sage Publications.

Cómo citar este artículo: Tabarquino, R. (2021). Consolidación legal y regulatoria del servicio público de las comunicaciones en Colombia 1847-2020. Tendencias, 23(1), 395-422. https://doi.org/10.22267/rtend.222301.195 